\title{
Dinâmica populacional de Bradybaena similaris (Férussac) (Molusca: Bradybaenidae) em ambiente protegido de borbulheiras cítricas
}

\author{
Luana Aparecida Castilho Maro ${ }^{1}$, Eduardo Rodrigues Hickel ${ }^{1} \&$ Marcelo Mendes Haro $^{1}$
}

\section{RESUMO}

A flutuação populacional do caracol Bradybaena similaris (Férussac) (Molusca: Bradybaenidae) em ambiente protegido de borbulheiras cítricas no Estado de Santa Catarina, Brasil, não é conhecida. Isto dificulta o desenvolvimento de novas estratégias para o manejo da praga, como também a racionalização da aplicação de moluscicidas para controle. Desta forma, objetivou-se monitorar a ocorrência de caracois nas plantas e no solo visando conhecer a flutuação populacional, correlacionando-a com a prática de manejo do solo desse ambiente que consiste em depositar sobre o solo o material vegetal proveniente da retirada de ramos porta-borbulhas. O procedimento amostral constou da inspeção semanal de armadilhas de solo e coleta com pano de batida, com registro das contagens de indivíduos por classe de tamanho, em solo mantido limpo ou com cobertura de folhas. A flutuação populacional de $B$. similaris, por estrato etário e classe de manejo de solo, seguiu um mesmo padrão. Houve ocorrência de mais indivíduos de julho a setembro e de janeiro a março, com nítida sobreposição de gerações. Nos períodos de outubro a dezembro e de abril a junho/julho, os caracois foram pouco detectados nos procedimentos amostrais. Conclui-se que a flutuação populacional de $B$. similaris, por estrato etário e classe de manejo de solo, seguiu um mesmo padrão, com ocorrência de mais indivíduos de julho a setembro e de janeiro a março; sendo que o manejo do solo não teve efeito sobre a população amostrada sobre as plantas.

Termos de indexação: caracol de jardim, ecologia de populações, manejo de pragas, Citrus.

\section{Population dynamics of Bradybaena similaris (Férussac) (Molusca: Bradybaenidae) in a citrus bud nursery}

\section{SUMMARY}

The Asian trampsnail, Bradybaena similaris (Férussac) (Molusca: Bradybaenidae), population dynamics in citrus bud nursery in Santa Catarina State, Brazil, is still unknown. This makes difficult the development of new strategies for pest management as well as the timing of molluscicides for control. The aim of this study was monitoring the trampsnail occurence on plants and soil to know its population dynamics and correlating it with the practice of soil management of this environment. The sampling procedure consisted of weekly inspection of soil traps and plant tapping, recording the counts of individuals by size class, in soil kept clean or covered with leaves. Population fluctuation of $B$. similaris by age group and soil management class followed the same pattern. More individuals

\footnotetext{
${ }^{1}$ Estação Experimental de Itajaí, Empresa de Pesquisa Agropecuária e Extensão Rural de Santa Catarina, Itajaí, SC, Brazil 
occurred from July to September and from January to March, with clear generational overlap. From October to December and from April to June / July, snails were poorly detected in the sampling procedures. It is concluded that the population fluctuation of $B$. similaris, by age stratum and soil management class, followed the same pattern, with more individuals occurring from July to September and from January to March; the soil management had no effect on the population sampled on the plants.

Index terms: Asian trampsnail, population ecology, pest management, Citrus.

\section{INTRODUÇÃO}

Os citros são propagados vegetativamente por enxertia, principalmente via borbulhia, e este método favorece o acúmulo de fitopatógenos transmissíveis, tais como vírus, viroides e bactérias, que podem permanecer em estado latente por vários anos. Dessa forma, é necessário que as mudas sejam produzidas a partir de material básico retirado de plantas matrizes ou borbulheiras obtidas e manejadas adequadamente (Carvalho et al., 2005). De acordo com a legislação federal vigente (Instrução Normativa $\mathrm{n}^{\circ} 48$, de 24 de setembro de 2013) (Brasil, 2013), que estabelece normas de produção, comercialização de material propagativo de citros, bem como seus padrões de identidade e qualidade, as borbulheiras certificadas deverão ser mantidas em ambiente protegido com tela de malha antiafídica para impedir a entrada de insetos vetores de fitopatógenos.

A infestação do ambiente protegido de cultivo de borbulheiras cítricas pelo caracol Bradybaena similaris (Férussac) (Molusca: Bradybaenidae) tem sido frequente em Santa Catarina. O solo predominante úmido nesse ambiente de borbulheiras no chão, associado à presença de vegetação ou matéria vegetal morta na superfície, que retorna ao solo após a retirada dos ramos porta-borbulhas, e à ausência de inimigos naturais, propicia altas populações deste caracol (Hickel et al., 2019). Nas borbulheiras, os indivíduos se alimentam das folhas causando pequenas perfurações no limbo foliar. Dependendo do nível de infestação, a perda de área foliar acarreta menor crescimento dos ramos, de onde são retiradas as borbulhas. Além disso, essa espécie tem importância sanitária, pois os indivíduos são hospedeiros intermediários de helmintos parasitas de animais e do homem (Araujo, 1989; Junqueira et al., 2003).

Bradybaena. similaris é o caracol de jardim, uma espécie asiática invasiva que ocorre por todo o Brasil (Almeida \& Bessa, 2001; Junqueira et al., 2003; Carvalho et al., 2008). Essa espécie habita os cultivos hortícolas, podendo eventualmente ser praga de hortaliças folhosas (Souza, 1999; Lutinski et al., 2016). Os indivíduos se alimentam de folhagem e matéria vegetal morta e vivem em média
145 dias, mas a longevidade pode alcançar até 480 dias. A atividade reprodutiva (idade adulta) se inicia entre os 78 e 160 dias, quando os caracois atingem diâmetro de concha superior a $8 \mathrm{~mm}$. Os ovos são postos agrupados, numa proporção variável de 3 a 115 ovos por postura e incubam por 24 dias, com $80 \%$ de viabilidade (Almeida \& Bessa, 2001; Carvalho et al., 2008; Junqueira et al., 2008).

Nesse ambiente de produção de borbulhas cítricas, algumas variáveis ambientais são mantidas sob controle, notadamente a precipitação e umidade do solo, que são providas pelo sistema de irrigação. A temperatura ambiente também sofre alteração, devido à cobertura plástica da casa de vegetação, porém a sazonalidade estacional é mantida.

Embora $B$. similaris esteja presente no ambiente de cultivo das borbulheiras cítricas e atinja altas populações, a variação sazonal dessa população não é conhecida. Também se desconhece a influência de manejos culturais executados nas borbulheiras, notadamente o manejo de solo, sobre a população de caracois. Essas informações são fundamentais para o planejamento de ações de controle da espécie, inclusive para a adoção de alternativas de tratos culturais, dentro de um programa de manejo integrado de pragas (Flint \& Van Den Bosch, 1981). Assim, foi objetivo desta pesquisa monitorar a ocorrência de caracois presentes nas plantas e no solo visando conhecer a flutuação populacional no decorrer do ano, correlacionando-a com a prática de manejo do solo (solo coberto por resíduos vegetais e solo limpo) no ambiente protegido de borbulheiras cítricas cultivadas no chão.

\section{MATERIAL E MÉTODOS}

A pesquisa foi conduzida na casa-de-vegetação telada que abriga as borbulheiras cítricas em solo tipo argiloso da Epagri, na Estação Experimental de Itajaí (EEI) no período de junho de 2017 a agosto de 2018. As plantas estão dispostas em linhas em espaçamento de $70 \mathrm{~cm}$ entre linhas e $30 \mathrm{~cm}$ entre plantas, sendo três linhas por cultivar. O ensaio compreendeu quatro parcelas de $8 \times 19 \mathrm{~m}$ cada, 


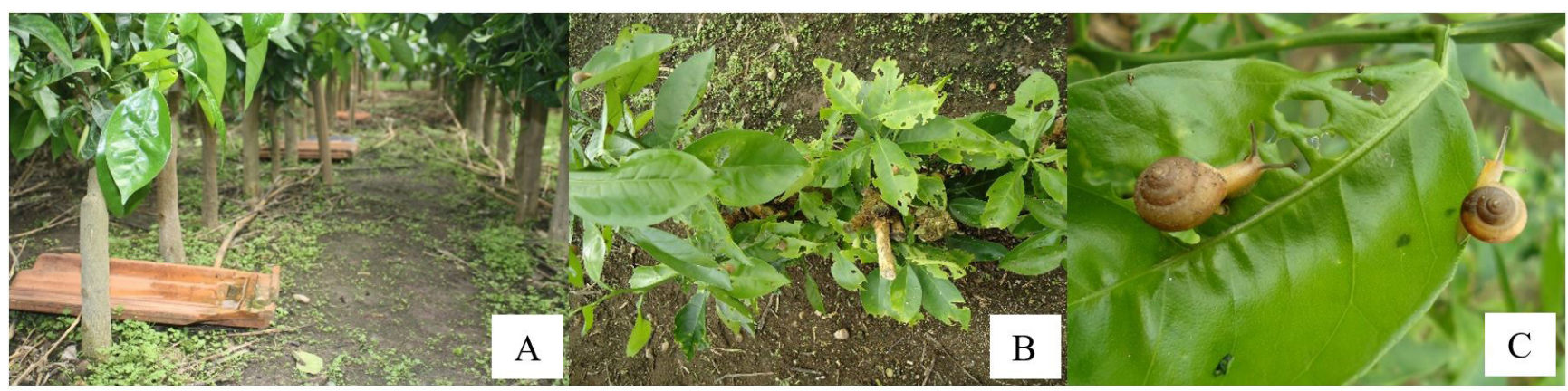

Figura 1. Vista geral da parcela com três armadilhas representadas por telhas cerâmicas do tipo francesa em solo mantido limpo mediante capinas manuais (A). Ramagem de plantas utilizadas para a amostragem dos caracois (B). B. similaris no estádio adulto (à esquerda) e juvenil II (à direita) alimentando-se do limbo foliar de plantas cítricas (C).

sendo que em duas parcelas o solo foi mantido limpo, mediante capinas manuais e nas outras duas o solo foi mantido coberto com o depósito superficial das folhas dos ramos extraídos para a obtenção das borbulhas. Esse depósito de folhas foi iniciado no mês de outubro de 2017 com a primeira retirada de ramos das plantas borbulheiras.

Armadilhas no solo, constituídas por uma telha cerâmica do tipo francesa sobre o solo, e amostragem nas plantas, usando pano de batida $\left(1 \mathrm{~m}^{2}\right)$, foram empregadas na amostragem populacional. Cada parcela recebeu três armadilhas de solo (Figura 1A), regularmente espaçadas e, a cada amostragem, o pano-de-batida também era usado próximo a esses pontos. Este era estendido no chão, na entrelinha de plantas, e a ramagem de seis plantas era sacudida com cabos de vassoura sobre o pano (Figura 1B). Foram estabelecidos dias para contagem semanal sempre no período da manhã, anotando-se o número de caracois (Figura 1C) de acordo com três classes de diâmetro da concha (idade) (Carvalho et al., 2008), a saber: juvenil I (até $4 \mathrm{~mm}$ ), juvenil II (de 4 a $8 \mathrm{~mm}$ ) e adulto (acima de $8 \mathrm{~mm}$ ). Após as contagens, os indivíduos foram liberados novamente no ambiente para não haver exclusão de caracois na população original e, consequentemente, não interferir no ciclo biológico da espécie. Com o registro das contagens estabeleceu-se a flutuação populacional, para cada estrato etário, correlacionando-as com a prática do manejo de solo. As diferenças entre manejo de solo e método de amostragem foram determinadas pela significância do teste de Qui-quadrado para as médias mensais de coleta de caracois, executando-se a rotina 'teste.qui' em planilha eletrônica Excel.

\section{RESULTADOS E DISCUSSÃO}

A flutuação populacional de $B$. similaris, por estrato etário e classe de manejo de solo, seguiu um mesmo padrão,
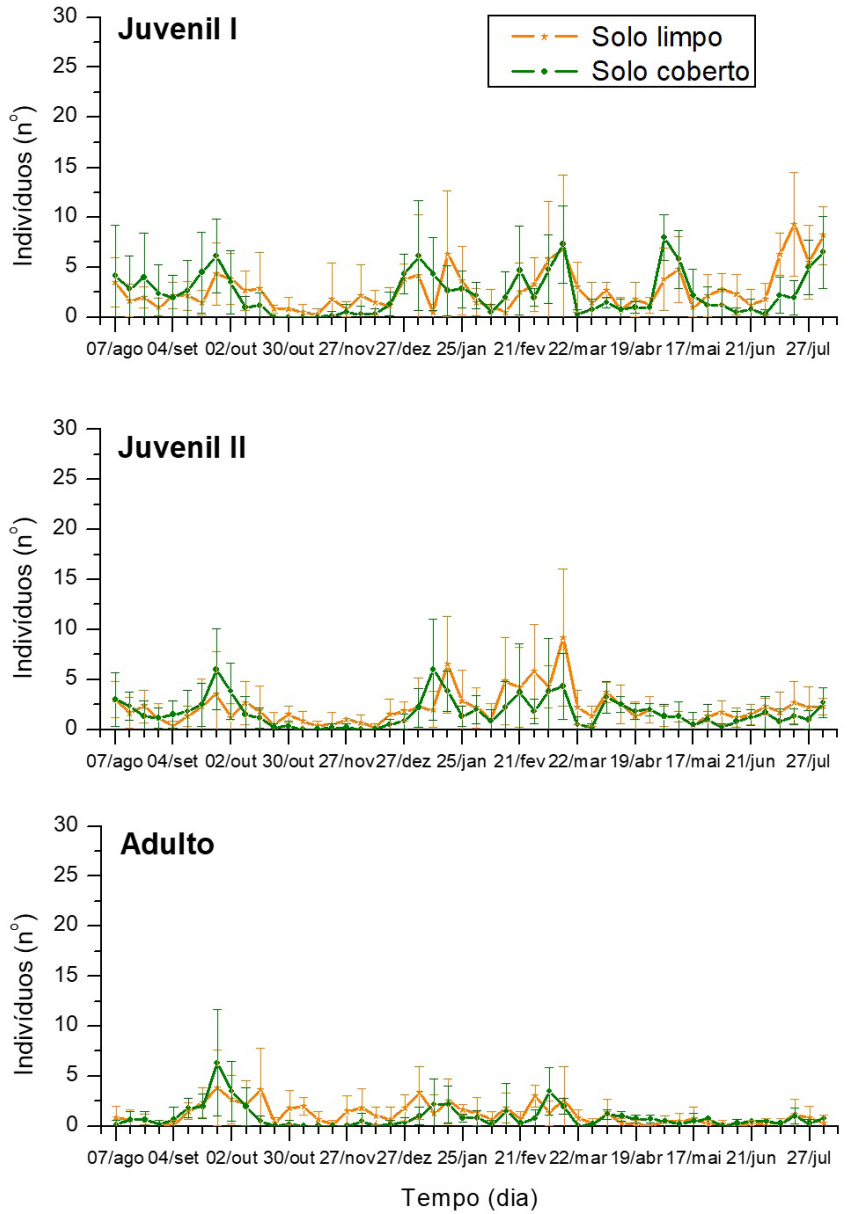

Figura 2. Flutuação populacional de $B$. similaris em ambiente protegido de borbulheiras cítricas. Número médio de indivíduos amostrados nas plantas, com pano de batida, e respectivo desvio padrão (linhas verticais). Itajaí, SC, 2017/2018.

embora tenha havido alta variabilidade nas contagens de indivíduos, nas diferentes datas amostrais, tanto na amostragem nas plantas quanto no solo (Figuras 2 e 3 ). 
Houve ocorrência de mais indivíduos de julho a setembro e de janeiro a março, com nítida sobreposição de gerações, ou seja, os três estratos etários ocorrendo concomitantemente (Knell, 1998). Sobreposição de gerações também ocorre nas populações de Bradybaena fruticum (Müller) em ambientes naturais na Polônia (Proćków et al., 2012).

Nos períodos de outubro a dezembro e de abril a junho/julho, os caracois foram pouco detectados nos procedimentos amostrais. Isso necessariamente não traduz a ausência de indivíduos no ambiente ou a sucessão de ciclos reprodutivos, pois também ocorreu simultaneamente nos três estratos etários. Tendo em vista que a população de $B$. similaris já estava estabelecida há vários anos no ambiente em estudo, é plausível que já estivesse em equilíbrio entre aporte e perda de indivíduos, dentro da capacidade de suporte do ambiente (Staikou et al., 1990). Assim, neste caso, a falta de detecção dos caracois
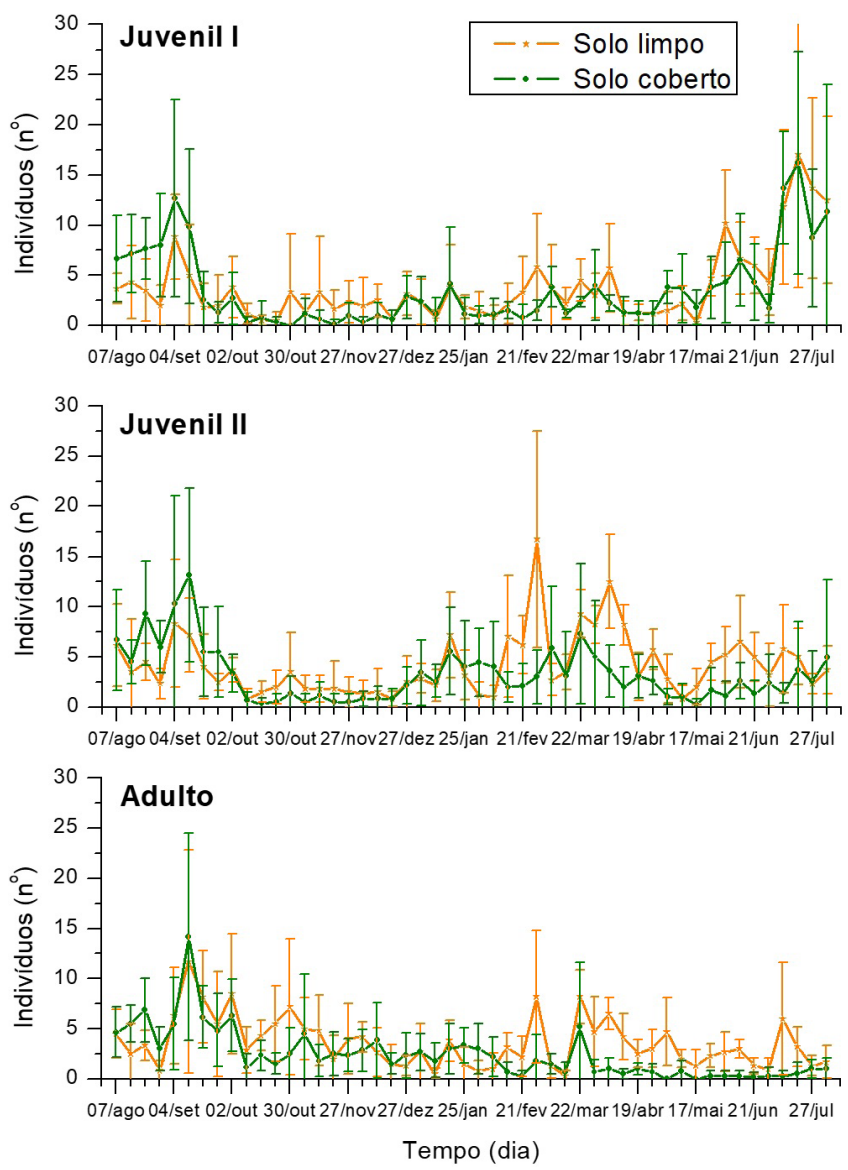

Figura 3. Flutuação populacional de $B$. similaris em ambiente protegido de borbulheiras cítricas. Número médio de indivíduos amostrados no solo, com armadilha de telha cerâmica, e respectivo desvio padrão (linhas verticais). Itajaí, SC, 2017/2018 pode ser atribuída a períodos de inatividade dos mesmos (Greenwood et al., 2019).

Junqueira et al. (2003), D’Ávila et al. (2004) e Almeida \& Afonso-Neto (2015), estudando o comportamento de B. similaris, reportam que os indivíduos têm períodos prolongados de inatividade e que isso pode estar relacionado à preparação para a atividade reprodutiva ou a estresses ambientais. O caracol B. fruticum hiberna por quatro meses, enterrado na camada superficial do solo, e permanece inativo, em estivação, durantes períodos secos do ano. Dessa forma, na flutuação populacional desta espécie, também é comum dois períodos anuais de pouca observação de indivíduos, especialmente quando ocorrem estiagens no verão (Staikou et al., 1990).

A inatividade dos caracois em novembro e dezembro foi mais uniforme e os indivíduos se ausentaram das plantas cítricas (Figura 2). Na população amostrada no solo (Figura 3), os adultos mantiveram certa atividade durante esse período. Adultos de $B$. similaris são normalmente mais ativos que os juvenis, tendo em vista a busca de parceiros e locais para nidificação, que ocorre no solo (Junqueira et al., 2003). A falta de atividade dos juvenis, nesse período, pode estar relacionada às etapas de crescimento (Staikou et al., 1990). Os caracois B. similaris são sensíveis a variações de umidade do solo e rapidamente entram em inatividade em períodos secos (D'Ávila et al., 2004), contudo, no ambiente de estudo, este fator estava sobre controle e estabilizado pela irrigação intermitente.

O segundo período de inatividade coincide com o de baixas temperaturas e diminuição da incidência de luz solar.Esses fatores não tiveram muita influência nos caracois mais jovens, mas foram sentidos pelos adultos. A reprodução de $B$. similaris ocorre primordialmente no verão. Assim, durante o inverno, os adultos restringem a atividade para se prepararem para o próximo ciclo reprodutivo (Staikou et al., 1990; Proćków et al., 2012). Não obstante, não há registros de $B$. similaris em hibernação no Brasil (Staikou et al., 1990; D’Ávila et al., 2004).

Maior número de juvenis I (caracois menores) foi registrado a partir de julho, principalmente nas amostragens no solo (Figura 3). Isso provavelmente resulta da atividade reprodutiva dos adultos durante o verão, com aporte de novos indivíduos na população (Staikou et al., 1990; Proćków et al., 2012). Nesse caso, embora a incubação de ovos em laboratório finde em 24 dias (Junqueira et al., 2008; Oliveira et al., 2008), em campo pode demorar mais tempo. Almeida \& Bessa (2001) verificaram que a incubação dos ovos sofre influência da temperatura, prolongando o período sob temperaturas mais baixas. 
Tabela 1. Número total médio de B. similaris amostrado nas plantas (pano-de-batida) e no solo (telha cerâmica) em três estratos etários, em função do manejo do solo. Itajaí, SC, 2017/2018

\begin{tabular}{|c|c|c|c|c|c|c|}
\hline \multirow{3}{*}{ Local } & \multicolumn{6}{|c|}{ Estrato etário } \\
\hline & \multicolumn{2}{|c|}{ Juvenil I } & \multicolumn{2}{|c|}{ Juvenil II } & \multicolumn{2}{|c|}{ Adulto } \\
\hline & Solo limpo & Solo coberto & Solo limpo & Solo coberto & Solo limpo & Solo coberto \\
\hline Planta & 138,2 & 122,0 & 108,2 & 84,3 & 59,0 & 103,3 \\
\hline Solo & 193,3 & 179,7 & 210,3 & 162,2 & 181,7 & 303,0 \\
\hline $\mathrm{p}_{\gamma}^{2}$ & 0,002 & 0,001 & $<0,001$ & $<0,001$ & $<0,001$ & $<0,001$ \\
\hline
\end{tabular}

A mortalidade de caracois $B$. similaris é gradual no tempo (Almeida \& Bessa, 2001; Carvalho et al., 2008; Junqueira et al., 2008), de modos que não ficou caracterizado, na flutuação populacional, períodos de maior perda de indivíduos. Isso corrobora a suposição que a população já estava estabilizada no ambiente quando do início do estudo.

O manejo do solo, limpo ou coberto com folhas, não teve efeito sobre a população de $B$. similaris amostrada sobre as plantas (Figura 2), para os três estratos etários $\left(\mathrm{p}_{\chi}^{2}=0,32\right.$ para juvenil $\mathrm{I} ; \mathrm{p}_{\chi}^{2}=0,09$ para juvenil II e $\mathrm{p}_{\chi}^{2}=0,15$ para adulto). O mesmo se sucedeu para amostragem de juvenis I no solo $\left(\mathrm{p}_{\chi}^{2}=0,48\right)$. Já para juvenis II $\left(\mathrm{p}_{\chi}^{2}=0,01\right)$ e caracois adultos $\left(\mathrm{p}_{\chi}^{2}=0,001\right)$, as contagens no solo limpo foram maiores. Era esperado que a cobertura do solo com folhas proporcionasse alimentação mais farta aos indivíduos e, em consequência, um aumento populacional pela maior fecundidade. Isso aparentemente não foi detectado no intervalo de tempo da pesquisa. A maior contagem de juvenis II e adultos no solo limpo certamente está relacionada ao abrigo para estivação, proporcionado pela umidade embaixo da telha de cerâmica (D’Ávila et al., 2004). Esse refúgio preferencial tornou-se menos atrativo num solo inteiramente mais úmido pelo efeito da cobertura de folhas.

Embora não fosse escopo principal da pesquisa, o número de $B$. similaris amostrado no solo foi maior que aquele amostrado nas plantas, independente do manejo do solo, em todos os estratos etários (Tabela 1). Isso torna a armadilha de solo, na forma de uma telha cerâmica, um aparato mais adequado para o monitoramento populacional. Após serem derrubados das plantas, o retorno dos caracois é lento, devido a sua baixa mobilidade no ambiente das borbulheiras cítricas (Hickel et al., 2019). Assim, o intervalo semanal das contagens não propiciou tempo hábil para a re-colonização das plantas entre as amostragens. Com o passar do tempo e pelo relativo pequeno tamanho das parcelas, eventualmente algumas plantas foram amostradas repetidamente.
Considerando o manejo do ambiente protegido e a produção de borbulhas cítricas, o planejamento de medidas de controle de $B$. similaris deverá estar voltado para o período de julho a setembro, anterior à entrada em inatividade dos indivíduos. Isso provavelmente reduzirá a população verificada entre janeiro e abril. Outro aspecto a considerar, é que esse controle é feito primordialmente com o uso de iscas alimentares tóxicas que contêm fosfato férrico na composição, as quais devem ser ofertadas nos períodos de atividade dos indivíduos e dispostas no solo

\section{CONCLUSÕES}

A flutuação populacional de B. similaris, por estrato etário e classe de manejo de solo, seguiu um mesmo padrão, com ocorrência de mais indivíduos de julho a setembro e de janeiro a março; sendo que o manejo do solo não teve efeito sobre a população amostrada sobre as plantas.

\section{AGRADECIMENTOS}

Aos acadêmicos de agronomia Rubiane Pellozo Alberti, Sérgio Francisco Bervanger e Vanessa Winter Forest, pelo auxílio nas amostragens.

À Fundação de Amparo à Pesquisa e Inovação do Estado de Santa Catarina (Fapesc) e Financiadora de Estudos e Projetos (Finep) pelo aporte financeiro.

\section{REFERÊNCIAS}

Almeida, M. N., \& Afonso-Neto, I. S. (2015). O gastrópode Bradybaena similaris (Férussac, 1821) (Pulmonata, Xanthonychidae) como modelo experimental para estudo em laboratório. Acta Biomedica Brasiliensia, 6(2), 25-41. http://dx.doi.org/10.18571/acbm.083. 
Almeida, M. N., \& Bessa, E. C. A. (2001). Estudo do crescimento e da reprodução de Bradybaena similaris (Férussac) (Mollusca, Xanthonychidae) em laboratório. Revista Brasileira de Zoologia, 18(4), 1115-1122.

Araujo, J. L. B. (1989). Moluscos de importância econômica no Brasil. I. Xanthonychidae: Bradybaena similaris (Férussac, 1821), (Mollusca, Gastropoda, Pulmonata, Stylommatophora). Revista Brasileira de Zoologia, 6(4), 583-592. http://dx.doi.org/10.1590/ S0101-81751989000400001.

Brasil. Ministério da Agricultura, Pecuária e Abastecimento. (2013, 24 de setembro). Instrução Normativa n. 48. Normas de Produção e Comercialização de Material de Propagação de Citros - Citrus spp., Fortunella spp., Poncirus spp., e seus híbridos, bem como seus padrões de identidade e de qualidade. Brasília, DF: Diário Oficial da República Federativa do Brasil, seção 1, p. 38.

Carvalho, C. M., Bessa, E. C. A., \& D’Ávila, S. (2008). Life history strategy of Bradybaena similaris (Fèrussac, 1821) (Mollusca, Pulmonata, Bradybaenidae). Molluscan Research, 28(3), 171-174.

Carvalho, S. A., Graf, C. C. D., \& Violante, A. R. (2005). Produção de material básico e propagação. In D. Mattos Junior, J. D. De Negri, R. M. Pio, \& J. Pompeu Junior (Eds.), Citros (pp. 279-316). Campinas: Instituto Agronômico e Fundag.

D’Ávila, S., Dias, R. J. P., Bessa, E. C. A., \& Daemon, E. (2004). Resistência à dessecação em três espécies de moluscos terrestres: aspectos adaptativos e significado para o controle de helmintos. Revista Brasileira de Zoociencias, 6(1), 115-127. Recuperado em 8 de junho de 2020, de https://periodicos. ufjf.br/index.php/zoociencias/article/ view/24209/13477

Flint, M. L., \& Van Den Bosch, R. (1981). Introduction to integrated pest management. New York: Plenum Press

Greenwood, D. J., Hall Junior, R. O., Tibbets, T. M., \& Krist, A. C. (2019). A precipitous decline in an invasive snail population cannot be explained by a native predator. Biological Invasions, 22, 363-378. http://dx.doi.org/10.1007/ s10530-019-02093-8.

Hickel, E. R., Maro, L. A. C., \& Haro, M. M. (2019). Identificação, estimativa populacional e deslocamento de caracois em borbulheiras de plantas cítricas. Revista de Estudos Ambientais, 21(1), 29-37.

Junqueira, F. O., Arévalo, E. G., \& Bessa, E. C. A. (2008). Influência do substrato sobre aspectos do ciclo de vida de Bradybaena similaris (Férussac, 1821) (Mollusca, Bradybaenidae), sob condições de laboratório. Revista Brasileira de Biociências, 6(4), 347-354. Recuperado em 8 de junho de 2020, de http://www.ufrgs.br/seerbio/ ojs/index.php/rbb/article/view/962

Junqueira, F. O., D’Ávila, S., Bessa, E. C. A., \& Prezoto, F. (2003). Ritmo de atividade de Bradybaena similaris (Férussac, 1821) (Mollusca, Xanthonychidae) de acordo com a idade. Revista de Etologia, 5(1), 41-46.

Knell, R. J. (1998). Generation cycles. Trends in Ecology \& Evolution, 13(5), 186-190. http://dx.doi.org/10.1016/ S0169-5347(97)01321-9.

Lutinski, J. A., Bonfante, F. P., Busato, M. A., \& Guarda, C. (2016). Plant extracts, metaldehyde and saline solutions on the population control of Bradybaena similaris. Ciência Rural, 46(8), 1316-1321. http://dx.doi. org/10.1590/0103-8478cr20151140.

Oliveira, C. S., Vasconcellos, M. C., \& Pinheiro, J. (2008). The population density effects on the reproductive biology of the snail Bradybaena similaris (Férussac, 1821) (Mollusca, Gastropoda). Brazilian Journal of Biology = Revista Brasileira de Biologia, 68(2), 367-371.

Proćków, M., Kúznik-Kowalska, E., \& Lewandowska, M. (2012). Differences in population dynamics of Bradybaena fruticum (O.F. Müller, 1774) (Gastropoda: Pulmonata: Bradybaenidae) in a submontane and lowland area of Poland. Animal Biology, 62(4), 451-462. http://dx.doi. org/10.1163/157075612X650131.

Souza, J. L. (1999). Cultivo orgânico de hortaliças: brócolis, couve-flor e repolho. Viçosa: CPT.

Staikou, A., Lazaridou-Dimitriadou, M., \& Pana, E. (1990). The life cycle, population dynamics, growth and secondary production of the snail Bradybaena fruticum (Müller, 1774) (Gastropoda Pulmonata) in northern Greece. The Journal of Molluscan Studies, 56(2), 137-146. http:// dx.doi.org/10.1093/mollus/56.2.137.

Recebido: Junho 08, 2020

Aceito: Janeiro 26, 2021

Como citar: Maro, L. A. C., Hickel, E. R., \& Haro, M. M. (2020). Dinâmica populacional de Bradybaena similaris (Férussac) (Molusca: Bradybaenidae) em ambiente protegido de borbulheiras cítricas. Citrus Research \& Technology, 41, e1062. https://doi.org/10.4322/crt.22020 